\title{
Awareness, Acceptance and Attitude on HIV/AIDS: \\ Perspectives of Students in a University
}

\author{
Mauro Allan P. Amparado \\ mapamparado@gmail.com \\ Director, Community Awareness, Relations \& Extension Services \\ University of Cebu Lapu-Lapu and Mandaue \\ Mandaue City, Cebu, Philippines
}

\section{Abstract}

This study ascertained the awareness, acceptance and attitude on HIV/AIDS of students in a university. The findings served as basis for a proposed HIV/AIDS education program. This study employed the mixed method design. It was conducted at UCLM, Mandaue City, Cebu, Philippines. Quantitatively, the study utilized 400 students who visited the medical clinic from July 1 , 2018 to August 31, 2018. The survey consists of 50 items, cafeteria questionnaire. Qualitatively, ten informants were interviewed for one hour, in four consecutive Saturdays. Using an unstructured interview, common themes that emerged from the one-on-one interview was analyzed.

Findings revealed that majority of the respondents were male (74.5\%), 18 to 20 years old $(78 \%)$, from the College of Marine Transportation (43.5\%), $1^{\text {st }}$ year students (56\%), and Roman Catholics (98\%). In terms of level of knowledge on HIV/AIDS, the table shows that majority of the respondents have excellent knowledge on this disease condition.

But based on the item analysis, the following questions had the highest number of wrong answers: confirmatory test for HIV/AIDS (80.5\%); phase where symptoms are evident such as rash, cough, malaise, night sweats and lymphadenopathy (76.5\%); phase with symptoms including lymphadenopathy, diarrhea, oral 
candidiasis, weight loss, fatigue, skin rash and fever (65\%); phase when test is positive for HIV antigens but no symptoms (64\%); and continent with the most number of HIV infections (61\%). Most of these questions pertain to the pathophysiology of the disease.

Although all ten informants confirmed that they will accept a person living with HIV, if they meet one, there is disparity between this acceptance and the way they respond to a scenario. In the scenario where they are eating with the PLWHIV, most of the informants were hesitant to get food from trays where the PLWHIV got his food. Misconceptions were also noted regarding HIV as a disease condition.

\section{Introduction}

AIDS, or acquired immune deficiency syndrome, is caused by infection with the human immunodeficiency virus (HIV). This virus is transmitted by contact with body fluids containing the virus through sexual contact, including anal intercourse, through contaminated needles, during birth from an infected mother, or by receiving contaminated blood in a transfusion. The infection has 3 stages: the initial symptoms, a latency period, and full-blown AIDS. Initial symptoms include weakness, fever, night sweats, weight loss, and swollen lymph glands in the neck region. These symptoms mimic the flu and last only a few days. The latency period may last 5 to 10 years with no symptoms. Full-blown AIDS occur with the onset of opportunistic infections that can be fatal. Some such infections are pneumonia, skin cancer, diarrhea, tuberculosis, toxoplasma affecting the nervous system, and fungal infections in the lungs and throat (Rizzo, 2010).

Activation of the immune response indicates the occurrence of infection. Infection results from tissue invasion and damage by an infectious agent. There are two types of infectious responses: localized infections and systemic infections. Localized infections 
are limited to a defined area or single organ with symptoms that resemble inflammation (redness, tenderness, and swelling). Systemic infections affect the entire body and involve multiple organs. Both progress through four stages of infection.

The incubation period is the time interval between entry of an infectious agent in the host and the onset of symptoms. During this time period, the infectious agent invades the tissue and begins to multiply to produce an infection.

The prodromal stage is the time interval from the onset of nonspecific symptoms until specific symptoms of the infectious process begin to manifest. During this period, the infectious agent continues to invade and multiply in the host. A client may also be infectious to other persons in this time period.

The illness stage is when the client is manifesting specific symptoms of an infectious process. The period of time from the beginning of the disappearance of acute symptoms until the client returns to the previous state of health is called convalescent stage (Delaune \& Ladner, 2002).

The value of vaccination of immunocompromised clients is not completely understood. HIV-positive clients may receive the flu vaccine; however, they may require a second vaccine to gain protection. Persons who should not be vaccinated include those with a known hypersensitivity to eggs or other components of the vaccine and adults with an acute febrile illness. The vaccines are formulated annually based on worldwide surveillance data.

Pneumococcal vaccine is recommended for clients at increased risk of developing pneumonia, those with chronic illnesses or immunosuppression (such as HIV/AIDS), those living in special environments such as nursing homes or the American Indian population, and clients over the age of 65. HIV-positive 
clients can also receive the pneu2mococcal vaccination. Revaccination has been recommended for clients at 65 years of age (Potter \& Perry, 2005).

HIV/AIDS also affects the social life of an individual on how they interact with others and how other people interact with an infected person. Likewise, interacting with a person who has it, is quite different from the usual interaction. The affected person may feel rejected caused by stigma. It would affect the social interaction such as having less or avoiding having conversation, approaching, building relationship with one another, and others. It would also lessen the support system because some would prefer to stay away or avoid interacting with the person who has HIV/AIDS (Li, Wu, Wu, Sun, Cui \& Jia, 2006).

\section{Theoretical Background}

This study is anchored on Merle Mishel's Uncertainty in Illness. This theory has twelve assertions (Tomey \& Alligood, 2002):

1. Uncertainty occurs when a person cannot adequately structure or categorize an illness-related event because there is lack of sufficient cues.

2. Uncertainty can take the form of ambiguity, complexity, lack of or inconsistent information, and/or unpredictability.

3. As symptom pattern, event familiarity, and event congruence (stimuli frame) increase, uncertainty decreases.

4. Structure providers (credible authority, social support, and education) decrease uncertainty directly by promoting interpretation of events and indirectly by strengthening the stimuli frame.

5. Uncertainty appraised as danger prompts coping efforts directed at reducing the uncertainty and managing the emotional arousal generated by it. 
6. Uncertainty appraised as opportunity prompts coping efforts directed at maintaining the uncertainty.

7. The influence of uncertainty on psychological outcomes is mediated by the effectiveness of coping efforts to reduce uncertainty appraised as danger or to maintain uncertainty appraised as opportunity.

8. When uncertainty appraised as danger cannot be effectively reduced, coping strategies can be employed to manage the emotional response.

9. The longer uncertainty continues in the illness context, the more unstable the individual's previously accepted mode of functioning becomes.

10. Under conditions of enduring uncertainty, individuals may develop a new, probabilistic perspective on life, which accepts uncertainty as a natural part of life.

11. The process of integrating continual uncertainty into a new view of life can be blocked or prolonged by structure providers who do not support probabilistic thinking.

12. Prolonged exposure to uncertainty appraised as danger can lead to intrusive thoughts, avoidance, and severe emotional distress.

\section{Related Studies}

A number of studies have shown that students who have received comprehensive sex education in school delay initiation of sex, reduce the number of partners, and are more likely to use contraception when they do have sex. And while the use of condoms cannot guarantee protection against pregnancy and HIV transmission, condoms do reduce risk. Nevertheless, the controversy continues in many communities. The decision on what students should be taught about sex is made by local school boards and depends on "community standards" (Schneider, 2011). 
In another study, it assessed the incidence and spectrum of AIDS-defining opportunistic illnesses in the highly active antiretroviral therapy (cART) era. Eight thousand and seventy patients (baseline median age 38 years; median CD4 cell count 298 cells/ $\mu$ l experienced 2027 incident opportunistic illnesses during a median of 2.9 years of observation. During 1994-1997, 1998-2002, and 2003-2007, respectively, rates of opportunistic infections (per 1000 person-years) were 89.0, 25.2 and 13.3 and rates of opportunistic malignancies were $23.4,5.8$ and 3.0 ( $P$ for trend $<0.001$ for both).

Opportunistic illness rate decreases were similar for the subset of patients receiving cART. During 2003-2007, there were no significant changes in annual rates of opportunistic infections or opportunistic malignancies; the leading opportunistic illnesses (rate per 1000 person-years) were esophageal candidiasis (5.2), Pneumocystis pneumonia (3.9), cervical cancer (3.5), Mycobacterium avium complex infection (2.5), and cytomegalovirus disease (1.8); $36 \%$ of opportunistic illness events occurred at CD4 cell counts at least 200 cells/ $\mu$ (Buchacz, Baker, Palella, Chmiel, Lichtenstein, Novak, \& HOPS Investigators, 2010).

DuRant, Ashworth, Newman, McGill, Rabun, \& Baranowski, revealed in their study that $68 \%$ reported having received schoolbased AIDS education, but a lower proportion $(\geq 10 \%)$ the students were found to correctly answer 8 of 17 AIDS/HIV knowledge questions than those from a national comparison group. The mean was $12.8 \pm 3.1$ of 17 items answered correct. Lower AIDS/ HIV knowledge was associated with lower school grade (rho $=0.46 p \leq$ 0.0001 ); being African-American, Hispanic, or Native American $(p \leq 0.043)$; and never receiving school-based AIDS/HIV education $(p \leq 0.0001)$. Based on multivariate analysis of variance (ANOVA), only school-based AIDS/HIV education was a significant predictor $(p \leq 0.0001)$ of knowledge. Self-reported behavior change to avoid HIV exposure was associated with previous AIDS education ( $p \leq$ 
0.025 ), and older age (rho $=-0.14, p \leq 0.016$ ). When these variables were analyzed $w$ ith multiple regression, only AIDS/HIV education was found to be a significant predictor $\left(R^{2}=0.022\right)$ of behavior change. Based on regression analysis, AIDS/HIV knowledge level and school grade explained 5.3\% $(p \leq 0.004)$ of the variation in the degree of worry of exposure to HIV. In turn, school grade, degree of worry of HIV exposure, and AIDS/HIV knowledge level explained $10 \%$ of the variation in the adolescents' perceived chance of having the HIV infection. These findings indicate that many of these younger rural adolescents lacked adequate knowledge to make responsible decisions about AIDS/HIV risk behavior and possessed beliefs about HIV exposure that may increase their risk (1992).

\section{Objectives}

This study ascertained the awareness, acceptance and attitude on HIV/AIDS of students in a university. The findings served as basis for a proposed HIV/AIDS education program.

\section{Methods}

This study employed the mixed method design. It was conducted at UCLM, Mandaue City, Cebu, Philippines. Quantitatively, the study utilized 400 students who visited the medical clinic from July 1, 2018 to August 31, 2018. The survey consists of 50 items, cafeteria questionnaire. To interpret the awareness of the respondents, the following scales were used:

$\begin{array}{lll}40-50 & - & \text { Excellent } \\ 30-39 & - & \text { Very Good } \\ 20-29 & - & \text { Good } \\ 10-19 & - & \text { Fair } \\ 0-9 & - & \text { Poor }\end{array}$


Qualitatively, ten informants were interviewed for one hour, in four consecutive Saturdays. Using an unstructured interview, common themes that emerged from the one-on-one interview was analyzed.

\section{Results and Discussion}

Table 1 presents the profile of the respondents. Majority were male $(74.5 \%), 18$ to 20 years old $(78 \%)$, from the College of Marine Transportation (43.5\%), $1^{\text {st }}$ year students (56\%), and Roman Catholics (98\%). In terms of level of knowledge on HIV/AIDS, the table shows that majority of the respondents have excellent knowledge on this disease condition.

But based on the item analysis, the following questions had the highest number of wrong answers: confirmatory test for HIV/AIDS (80.5\%); phase where symptoms are evident such as rash, cough, malaise, night sweats and lymphadenopathy (76.5\%); phase with symptoms including lymphadenopathy, diarrhea, oral candidiasis, weight loss, fatigue, skin rash and fever (65\%); phase when test is positive for HIV antigens but no symptoms (64\%); and continent with the most number of HIV infections (61\%). Most of these questions pertain to the pathophysiology of the disease.

\section{Table 1. Profile of the Respondents}

\begin{tabular}{|l|c|c|}
\hline Profile & Frequency & Percentage \\
\hline Gender & & \\
\hline Female & 51 & 25.5 \\
\hline Male & 149 & 74.5 \\
\hline Total & 200 & 100 \\
\hline Age & & \\
\hline $18-20$ & 156 & 78 \\
\hline $21-23$ & 35 & 17.5 \\
\hline
\end{tabular}




\begin{tabular}{|l|c|c|}
\hline $24-26$ & 9 & 4.5 \\
\hline Total & 200 & 100 \\
\hline Department & & \\
\hline $\begin{array}{l}\text { College of Business \& } \\
\text { Accountancy }\end{array}$ & 2 & 1 \\
\hline College of Computer Studies & 30 & 15 \\
\hline College of Criminology & 10 & 5 \\
\hline College of Marine Engineering & 41 & 20.5 \\
\hline $\begin{array}{l}\text { College of Marine } \\
\text { Transportation }\end{array}$ & 87 & 43.5 \\
\hline College of Teacher Education & 30 & 15 \\
\hline Total & 200 & 100 \\
\hline Year Level & & \\
\hline $1^{\text {st }}$ year & 112 & 56 \\
\hline $2^{\text {nd }}$ year & 56 & 28 \\
\hline $3^{\text {rd }}$ year & 14 & 7 \\
\hline $4^{\text {th }}$ year & 18 & 9 \\
\hline Total & 200 & 100 \\
\hline Religion & & \\
\hline Roman Catholic & 196 & 98 \\
\hline Protestant & 4 & 2 \\
\hline Total & 200 & 100 \\
\hline Level of Knowledge & & \\
\hline Excellent & 94 & 48.5 \\
\hline Very Good & 79 & 39.5 \\
\hline Good & 20 & 10 \\
\hline Fair & 200 & 3.5 \\
\hline Total & & 100 \\
\hline
\end{tabular}


Table 2. Item Analysis

\begin{tabular}{|c|c|c|c|c|c|}
\hline $\begin{array}{c}\text { Question } \\
\text { No. }\end{array}$ & $\begin{array}{c}\text { Wrong } \\
\text { Answer }\end{array}$ & $\begin{array}{c}\text { Proportion } \\
\%\end{array}$ & $\begin{array}{c}\text { Question } \\
\text { No. }\end{array}$ & $\begin{array}{c}\text { Wrong } \\
\text { Answer }\end{array}$ & $\begin{array}{c}\text { Proportion } \\
\%\end{array}$ \\
\hline 1 & 32 & 16 & 16 & 37 & 18.5 \\
\hline 2 & 54 & 27 & 17 & 79 & 39.5 \\
\hline 3 & 51 & 25.5 & 18 & 58 & 29 \\
\hline 4 & 11 & 5.5 & 19 & 36 & 18 \\
\hline 5 & 14 & 7 & 20 & 69 & 34.5 \\
\hline 6 & 42 & 21 & 21 & 27 & 13.5 \\
\hline 7 & 16 & 8 & 22 & 51 & 25.5 \\
\hline 8 & 11 & 5.5 & 23 & 122 & 61 \\
\hline 9 & 15 & 7.5 & 24 & 107 & 53.5 \\
\hline 10 & 95 & 47.5 & 25 & 161 & 80.5 \\
\hline 11 & 14 & 7 & 26 & 73 & 36.5 \\
\hline 12 & 27 & 13.5 & 27 & 36 & 18 \\
\hline 13 & 32 & 16 & 28 & 153 & 76.5 \\
\hline 14 & 39 & 19.5 & 29 & 128 & 64 \\
\hline 15 & 20 & 10 & 30 & 130 & 65 \\
\hline
\end{tabular}

Ten informants were interviewed guided by two scenarios. The interviews were conducted in four consecutive sessions of one-hour interview. It turned out in the interview that all of the informants claimed that they will accept a person living with HIV. They also shared that they will not discriminate a person if they discovered if he/she has HIV.

Ironically, when the informants were given a scenario, there seems to be a disparity between what they claim as acceptance. In this scenario, the informant is having lunch buffet with a person living with HIV (PLWHIV). Surprisingly, eight out of the ten informants shared that they will be hesitant to dine with the PLWHIV. Informant 1 shared, "I will not use the serving spoon that he/she used. I know that HIV cannot be transmitted through food, 
but I am not comfortable using the serving spoon he/she has used." Informant 5 shared, "I will move away from the line of the buffet if I knew that I will be next to a person living with HIV. I will get food once he/she is through getting food." Similarly, Informant 8 had the same sentiments. "I will be very observant of the food he/she takes and avoid getting from those food tray."

Some of the misconceptions from the informants that were extracted during the interview includes the following:

- HIV comes from animals. People were infected when they had intercourse with animals.

- Cross contamination is possible through sharing of food.

- It is primarily a disease of the Business Processing \& Outsourcing companies.

- HIV can be transmitted to other persons if the person living with HIV has poor hygiene habits.

- The virus that causes AIDS can be found on fruits. These fruits have been injected with blood infected with the virus.

\section{Conclusion}

Although all ten informants confirmed that they will accept a person living with HIV, if they meet one, there is disparity between this acceptance and the way they respond to a scenario. In the scenario where they are eating with the PLWHIV, most of the informants were hesitant to get food from trays where the PLWHIV got his food. Misconceptions were also noted regarding HIV as a disease condition. 


\section{Literature Cited}

Buchacz, K., Baker, R. K., Palella Jr, F. J., Chmiel, J. S., Lichtenstein, K. A., Novak, R. M., ... \& HOPS Investigators. (2010). AIDS-defining opportunistic illnesses in US patients, 1994-2007: a cohort study. Aids, 24(10), 1549-1559.

Delaune, S. C. \& Ladner, P. K. (2002). Fundamentals of Nursing: Standards \& Practice. $2^{\text {nd }}$ edition. Singapore: Delmar.

DuRant, R. H., Ashworth, C. S., Newman, C. L., Mcgill, L., Rabun, C., \& Baranowski, T. (1992). AIDS/HIV knowledge level and perceived chance of having HIV among rural adolescents. Journal of Adolescent Health, 13 (6), 499-505.

Li, L., Wu, S., Wu, Z., Sun, S. Cui, H. \& Jia, M. (2006). Understanding family support for people living with HIV/AIDS in Yunman, China. AIDS and Behavior, 10 (5), 509-517.

Potter, P. A. \& Perry, A. G. (2005). Fundamentals of Nursing. $6^{\text {th }}$ Edition. Singapore: Elsevier Pte. Ltd.

Rizzo, D. C. (2010). Fundamentals of Anatomy and Physiology. $3^{\text {rd }}$ edition. USA: Cengage Learning.

Schneider, M. J. (2011). Introduction to Public Health. $3^{\text {rd }}$ edition. Singapore: Jones and Bartlett Publishers 


\section{University of Cebu Lapu-Lapu and Mandaue}

\section{HIV/AIDS Questionnaire}

Instructions: For the profile, please check your gender, civil status, department, category, year level, and religion. For age and department, please write your age and department on the line. For the HIV/AIDS Test, please write the letter of your choice on the line before each number.

Gender: Male Female

Age:

Civil Status:

Single

Married

Single Parent

Separated

Widow

Category:

Student

Teaching staff

Non-teaching staff

Alumni

Course or Department:

Year Level:

Religion: 
1. What is the meaning of the acronym HIV?
A. Human Immune Virus
B. Host Immunodeficiency Virus
C. Human Immunodeficiency Virus

2. What is the meaning of acronym AIDS?
A. Acquired Immune Disease Syndrome
B. Acquired Immune Death Syndrome
C. Acquired Immune Deficiency Syndrome

3. What is the difference between HIV and AIDS?
A. There is no difference between HIV and AIDS
$B$. HIV is the virus and AIDS is the bacteria
C. HIV is the virus that causes AIDS and AIDS is the stage of infection

4. HIV can make a person ill because:
A. It makes the person gain weight
B. It attacks the immune system
C. It causes water retention on the body

5. Which body fluid contains the HIV if a person is infected?
A. Tears
B. Blood
C. Sweat

6. HIV is not present in what body fluid?
A. Breast milk
B. Sweat
C. Semen or vaginal fluid

7. What is meant by unprotected sex?
A. Kissing and cuddling with partner
B. Sex with condom
C. Sex without condom 
8. Which sector has the highest number of HIV infections?
A. LGBT
B. Children
C. People with disability

9. Which group is at risk of getting the HIV infection?
A. People who use condom during sex
B. People who share needles and syringes
C. People who hug and kiss

10. Which of the following statements is not true?
A. People infected with HIV/AIDS are asymptomatic until they die.
B. HIV lowers the body's resistance to disease and infection.
C. Persons infected with the HIV could get worse and develop to AIDS.

11. HIV is considered as:
A. Airborne disease
B. Waterborne disease
C. Blood borne disease

12. HIV may be transmitted through:
A. Blood and blood products
B. Kissing
C. Touching person with HIV

13. HIV may also be transmitted through:
A. Kissing
B. Mother to child upon delivery of baby
C. Sex with condom

14. Which statement is true? 

A. Persons infected with HIV/AIDS must stay in an isolated area and avoid social interaction.
B. Persons infected with HIV/AIDS should seeks immediate treatment and care.
C. Persons infected with HIV/AIDS are not allowed to work in order to prevent the spread of infection.

15. Refers to a thin layer of latex, polyutherane or polyisoprene worn over the penis during sex.
A. Plastic
B. Contraceptives
C. Condom

16. Which of the following will prevent HIV/AIDS infection?
A. Abstinence
B. Multiple sex partners
C. Sharing needles with friends

17. What is the medication for HIV/AIDS?
A. Antibacterial
B. Antifungal
C. Antiretroviral

18. Which statement is correct about condoms?
A. Condoms may be stored in your wallet.
B. Always check the expiration date of the condom wrapper or box.
C. Condom may be used with oil-based lubricants.

syringes?

19. Which statement is correct about disposing needles and

A. Dispose needles and syringes in a "sharps" container or a puncture-resistant plastic bottle. Bleach or laundry detergent bottles are good choices. 
B. Dispose sharps in milk cartons, plastic bags and garbage cans.

C. Keep the syringe and re-cap the needle.

20. Which statement is incorrect?

A. Persons infected with HIV must be isolated and should avoid socializing with other people.

B. Persons infected with HIV can prevent AIDS by taking the treatment as soon as possible.

C. Persons infected with HIV are susceptible to opportunistic infections.

21. What is the best way to avoid HIV/AIDS infection through blood transfusion?

A. Ask the blood donor if he/she has HIV/AIDS.

B. Blood must undergo series of tests for HIV/AIDS infection before the blood transfusion.

C. Avoid blood transfusion as much as possible.

22. HIV can be transmitted through:
A. Mosquito bite
B. Eating with persons infected with HIV
C. Breastmilk

infections.

23. In 2017, the continent with the most number of HIV
A. Asia
B. Africa
C. North America

24. HIV/AIDS attacks the immune system by destroying
A. Red blood cells
B. Bones
C. CD4 cells 
25. The confirmatory test for HIV/AIDS.
A. ELISA
B. Western Blot
C. Complete blood count

26. Which statement is correct about HIV/AIDS?
A. There is a vaccine for HIV/AIDS.
$B$. There is a cure for HIV/AIDS.
C. There is no cure for HIV/AIDS.

27. Which sector is the most vulnerable to HIV/AIDS?
A. Men having sex with women
B. Men having sex with men
C. Women having sex with women

28. Phase where symptoms are evident such as rash, cough, malaise, night sweats and lymphadenopathy.
A. Active phase
B. Asymptomatic
C. AIDS-related complex phase

29. Phase when test is positive for HIV antigens but no symptoms.
A. Active phase
B. Asymptomatic
C. AIDS-related complex phase

30. Phase with symptoms including lymphadenopathy, diarrhea, oral candidiasis, weight loss, fatigue, skin rash, and fever.
A. Active phase
B. AIDS phase
C. AIDS-related complex phase 
